\title{
Determine the Topology of Real Algebraic Surfaces
}

\author{
Jin-San Cheng, Xiao-Shan Gao and Ming Li \\ xgao@mmrc.iss.ac.cn, jcheng@amss.ac.cn, M.Li@cs.cardiff.ac.uk \\ Key Lab of Mathematics Mechanization, Institute of Systems \\ Science, AMSS Academia Sinica, Beijing 100080, China
}

\begin{abstract}
An algorithm is proposed to determine the topology of an implicit real algebraic surface in $\mathbb{R}^{3}$. The algorithm consists of four steps: surface projection, projection curve topology determination, space curve segmentation and surface patch composition, combination of surface patches and surface topology representation. The topology is represented by a set of surface patches, and each surface patch is presented by an ordered list of space curve segments. The relationship between the surface patches can be found by their space curve segments. Some examples show that our algorithm is effective.
\end{abstract}

Keywords: real algebraic surface, plane curve topology determination, space curve segmentation, surface patch, surface topology

\section{Introduction}

An implicit real algebraic surface(or curve, or hypersurface) $\mathcal{S}$ in $\mathbb{R}^{m}$ with degree $n$ is defined by $f\left(x_{1}, x_{2}, \cdots, x_{m}\right)=0$ where $f\left(x_{1}, x_{2}, \cdots, x_{m}\right) \in \mathbb{R}\left[x_{1}, x_{2}, \cdots, x_{m}\right]$ is a polynomial of degree $n$ and $\mathbb{R}$ the field of real number. What is the topology of $\mathcal{S}$ ? $\mathcal{S}$ is a set of discrete points on a line when $m=1$. When $m=2, \mathcal{S}$ is a plane algebraic curve. Algorithms to determine the topology of plane algebraic curve were proposed in (Hoon, 1996 and GonzalezVega and Necula, 2002). When $m=3$, the problem is more complex. The topology of $\mathcal{S}$ with $n=2$ is well known. They are some quadratic surfaces. But when $n \geq 3$, there are only some special surfaces whose topology can be determined.

Fortuna et al presented an algorithm (Fortuna etc. 2003) to determine the topology of non-singular, orientable real algebraic surface in projective space. But for general case, there is no existing algorithm to determine the topology of $\mathcal{S}$. Theoretically, the CAD method proposed by Collins can be used to determine information about the topology of an algebraic surface $[2,3]$. But, a complete algorithm is not given.

In this paper, we present an algorithm to determine the topology of $\mathcal{S}$ of $m=3, n \geq 3$. In the rest of this paper, we replace $f\left(x_{1}, x_{2}, x_{3}\right)=0$ with $f(x, y, z)=0$.

The basic idea of our algorithm is as follows. Project $\mathcal{S}: f(x, y, z)=0$ to plane and obtain a plane algebraic curve $\mathcal{C}: g(x, y)=0$. Analyze the topology of $\mathcal{C}$ in a finite box, get its singularities, segment the curve into plane curve segments and label its cells. Segment the space curve defined by $\{f(x, y, z)=0, g(x, y)=0\}$ into space curve segments. Compute 
the number of surface patches originating from each space curve segment in two cell bodies and register each surface patches using its boundary space curve segments. Combine surface patches if they having common critical space curve segment. In the end, we obtain a set of surface patches presented by boundary space curve segments and critical space curve segments of $\mathcal{S}$.

It is difficult to analyze the computing time of the algorithm. The most time-consuming part of the algorithm is that of obtaining $\mathcal{C}$, analyzing $\mathcal{C}$ and space curve segmentation.

This paper is divided into seven sections. The second section is to obtain projection curve of the surface. The third section presents an algorithm to determine the topology of the plane projection curve and getting some information which we need in the following sections, such as singularities, curve segments, cells, and so on. Space curve segmentation and surface patch composition are discussed in the fourth section. The fifth section discusses the combination of surface patches and the surface topology representation. The sixth section presents the main algorithm to obtain the topology of a given algebraic surface. Then we draw a conclusion in the last section.

\section{Projection curve of the surface}

In the following, we will show algorithms to determine topology of al algebraic surface $\mathcal{S}: f(x, y, z)=0$, where $f(x, y, z) \in \mathbb{Z}[x, y, z], \mathbb{Z}$ is the ring of integers.

In the section, our aim is to get the projection curve of the given surface $\mathcal{S}$. We will discuss how to obtain the projection curve of $\mathcal{S}$.

When $f(x, y, z)$ is irreducible, the discriminant of the surface is as follows.

$$
g(x, y)=\operatorname{Res}\left(f(x, y, z), \frac{\partial f(x, y, z)}{\partial z}, z\right)
$$

where $\operatorname{Res}\left(f(x, y, z), \frac{\partial f(x, y, z)}{\partial z}, z\right)$ is the resultant of $f(x, y, z)$ and $\frac{\partial f(x, y, z)}{\partial z}$

In order to fit the operation below and make the operation easily, that is, the discriminant obtained should be effective and efficient to determine the topology of $\mathcal{S}$, we should insure that

1. there is no point $P_{0}\left(x_{0}, y_{0}\right)$ on plane, which satisfies $f\left(x_{0}, y_{0}, z\right)=0$, it is clear that $P_{0}$ vanish on the discriminant;

2. the sum polynomial of terms with total degree of $f(x, y, z), \sum_{i+j+k=n} a_{i, j, k} * x^{i} * y^{j} * z^{k}$, has no factor who does not have variable $z$, where $n$ is total degree of $f(x, y, z)$.

An opportune surface is an irreducible algebraic surface $f(x, y, z)$ which satisfies the two cases.

If there exists line of $\mathcal{S}$ which is vertical to XY-plane, the number of the lines is finite. Otherwise, there is a cylinder in $\mathcal{S}$. It is in contradiction to the fact $\mathcal{S}$ is irreducible or $f(x, y, z)$ has three variables.

Otherwise, for case $1, f(x, y, z)$ has a line which is vertical to the XY-plane, we cannot compute the surface patches which intersect the line lifting from $P_{0}$. We can represent $f(x, y, z)$ as follows.

$$
f(x, y, z)=c_{k}(x, y) * z^{k}+c_{k-1} * z^{k-1}+\cdots+c_{0}(x, y),
$$


where $c_{i}(x, y) \in \mathbb{Z}[x, y]$ and $c_{k}(x, y)$ is nonzero polynomial. If $f(x, y, z)$ does not satisfy case 1 , we can derive that the variety $\left\{c_{0}(x, y)=0, c_{1}(x, y)=0, \cdots, c_{k}(x, y)=0\right\}$ has real $\operatorname{root}(\mathrm{s})$.

For example, given the following equation,

$$
f(x, y, z)=x^{2} * y^{2}+z^{2} * y^{2}+x^{2} * z^{2}-7 / 2 * x * y * z,
$$

we can derive $f(0,0, z)=0$ for all $z$, which means the line $\{x=0, y=0\}$ is on the surface. We don't know how many surface patches intersect the line. We can take a transformation of coordinate system to satisfy case 1 . If $c_{k}(x, y)$ is a constant, it is clear that case 1 holds.

For case 2, the asymptotic surface( a surface approaching a given surface arbitrarily closely) of $f(x, y, z)$ is vertical to XY-plane, we cannot easily to compute the number of surface patches originating from its corresponding space curve segments. We can represent $f(x, y, z)$ as follows.

$$
f(x, y, z)=\sum_{i+j+k=n} a_{i, j, k} * x^{i} * y^{j} * z^{k}+\sum_{i+j+k=n-1} a_{i, j, k} * x^{i} * y^{j} * z^{k}+\cdots+a_{0,0,0}
$$

Then we can derive the sum polynomial of terms with total degree of $f(x, y, z): \sum_{i+j+k=n} a_{i, j, k} *$ $x^{i} * y^{j} * z^{k}=T(x, y) * T_{0}(x, y, z)$.

For example, when $f(x, y, z)=x * z^{2}+y * z-1$, the sum polynomial of terms with total degree of $f(x, y, z)$ is $x * z^{2}, x=0$ is an asymptotic surface( plane) of the surface. The projection curve of the surface is $-4 * x^{2}-y^{2} * x=0$, but $f(0,0, z)=-1 \neq 0$, we cannot determine the boundaries of the surface patches which are approaching the plane $x=0$.

So we need to take a transformation of the coordinate system to avoid the two cases happening. For case 2, it is easy to do so. We can take the following transformation of coordinate system.

$$
\left(\begin{array}{l}
x \\
y \\
z
\end{array}\right)=\left(\begin{array}{lll}
1 & 0 & a \\
0 & 1 & b \\
0 & 0 & 1
\end{array}\right)\left(\begin{array}{l}
X \\
Y \\
Z
\end{array}\right),
$$

where $(x, y, z),(X, Y, Z)$ are any point in the old coordinate system and the new coordinate system respectively. We can choose any $a, b(a \neq 0, b \neq 0)$. Denote the corresponding surface in the new coordinate system as $F(X, Y, Z)$. As is well known, a transformation of coordinate system does not change the topology of a surface. So the surface $f(x, y, z)$ has the same topology with the surface $F(X, Y, Z)$. Represent $F(X, Y, Z)$ as follows.

$$
F(X, Y, Z)=C_{n}(X, Y) * Z^{n}+C_{n-1}(X, Y) * Z^{n-1}+\cdots+C_{0}(X, Y),
$$

where $C_{n}(X, Y)=\sum_{i+j+k=n} a_{i, j, k} * a^{i} * b^{j} * 1^{k}$. For case 1 , we need to find $a, b$ such that

$$
\left\{C_{n}(X, Y), C_{n-1}(X, Y), \cdots, C_{0}(X, Y)\right\}
$$

has no real root. If we can choose $a, b$ such that $C_{n}(X, Y)$ is a nonzero constant, it is OK. We can choose a rational point $(a, b)$ which is not on the curve $\sum_{i+j+k=n} a_{i, j, k} * x^{i} * y^{j} * 1^{k}=0$. Then two problems are solved.

For case 2, here is an example. For the surface $x * y * z-1=0$, we choose $a=$ $1, b=1$. The new surface which has a same topology with the surface $x * y * z-1=0$ 
is $F(X, Y, Z)=Z^{3}+(Y+X) * Z^{2}+X * Y * Z-1$. Its projection curve is $G(X, Y)=$ $27+6 * X^{2} * Y+6 * X * Y^{2}-X^{4} * Y^{2}+2 * X^{3} * Y^{3}-X^{2} * Y^{4}-4 * Y^{3}-4 * X^{3}$. There is no asymptotic surface projected in $G(X, Y) . F(X, Y, Z)$ is an opportune surface.

We will show you how to deal with the example: Equation 1. Represent $\mathcal{S}$ as follows.

$$
f(x, y, z)=\left(y^{2}+x^{2}\right) * z^{2}-7 / 2 * x * y * z+x^{2} * y^{2}=0 .
$$

The variety $\left\{y^{2}+x^{2},-7 / 2 * x * y, x^{2} * y^{2}\right\}$ has a common real root $\{x=0, y=0\}$. So we need to take a transformation of coordinate system. Follow the method presented above, we need to choose a rational number pair $(x, y)=(a, b)$, such that $\left(y^{2}+x^{2}\right) * 1^{2}+x^{2} * y^{2}$ is nonzero. We can choose $(a, b)=(1,1)$. The new surface is

$F(X, Y, Z)=3 * Z^{4}+4 * Y * Z^{3}+4 * X * Z^{3}-7 / 2 * Z^{3}+2 * Y^{2} * Z^{2}+2 * X^{2} * Z^{2}-7 / 2 * Y *$ $Z^{2}-7 / 2 * X * Z^{2}+4 * X * Y * Z^{2}-7 / 2 * X * Y * Z+2 * X * Y^{2} * Z+2 * X^{2} * Y * Z+X^{2} * Y^{2}$. And there is no number pair $\left(x_{0}, y_{0}\right)$ such that $F\left(x_{0}, y_{0}, Z\right)=0$. It is an opportune surface.

If $g(x, y)=0$ is reducible, factor it as follows.

$$
g(x, y)=g_{1}(x, y)^{n_{1}} \cdots g_{m}(x, y)^{n_{m}} .
$$

Let

$$
g_{0}(x, y)=g_{1}(x, y) \cdots g_{m}(x, y) .
$$

The two equation $g_{0}(x, y)=0$ and $g(x, y)=0$ express the same plane curve. So we only need to compute the topology of $g_{0}(x, y)=0$.

An opportune projection of an irreducible surface is a square-free polynomial which has same real zeros with the discriminant of the surface.

Follow the discussion above, we can derive the following algorithm to obtain the projection curve of a given irreducible surface $f(x, y, z)=0$.

Algorithm 1 The input is an irreducible polynomial $f(x, y, z)$. The output is an opportune projection of the surface $f(x, y, z)=0: g(x, y)=0$.

1. Represent $f(x, y, z)$ as $f(x, y, z)=c_{k}(x, y) * z^{k}+c_{k-1} * z^{k-1}+\cdots+c_{0}(x, y)$, check whether the variety $\left\{c_{k}(x, y), c_{k-1}, \cdots, c_{0}(x, y)\right\}$ has real root or not. If it has real $\operatorname{root}(\mathrm{s})$, go to 3 .

2. Represent $f(x, y, z)$ as $f(x, y, z)=\sum_{i+j+k=n} a_{i, j, k} * x^{i} * y^{j} * z^{k}+\sum_{i+j+k=n-1} a_{i, j, k} *$ $x^{i} * y^{j} * z^{k}+\cdots+a_{0,0,0}$, check whether $\sum_{i+j+k=n} a_{i, j, k} * x^{i} * y^{j} * z^{k}$ has a factor which does not have variable $z$. If it does not have this factor, go to 4 .

3. If there holds one of the two cases above, take the transformation of coordinate system as Equation (2), choose rational number pair $(a, b)$ such that $(a, b)$ is not a point of curve $\sum_{i+j+k=n} a_{i, j, k} * x^{i} * y^{j} * 1^{k}=0$, and compute the corresponding new surface $F(X, Y, Z)$ in the new coordinate system. Denote $F(X, Y, Z)$ still as $f(x, y, z)$.

4. Compute the discriminant of $f(x, y, z)$. Denote $g(x, y)=\operatorname{Res}\left(f(x, y, z), \frac{\partial f(x, y, z)}{\partial z}, z\right)$.

5. If $g(x, y)$ is irreducible, return it. Else, factor it as $g(x, y)=g_{1}(x, y)^{m_{1}} * g_{2}(x, y)^{m_{2}} *$ $\cdots * g_{t}(x, y)^{m_{t}}$, where $g_{i}(x, y)$ is irreducible. Still denote $g(x, y)=g_{1}(x, y) * g_{2}(x, y) *$ $\cdots * g_{t}(x, y)$ and return it. 
When $f(x, y, z)$ is reducible, the problem is more complex. Factor $f(x, y, z)$ we can obtain the following expression.

$$
f(x, y, z)=f_{1}(x, y, z)^{m_{1}} * \cdots * f_{n}(x, y, z)^{m_{n}},
$$

where each component $f_{i}(x, y, z)$ is irreducible. If one component only content variable $z$, it is some discrete points. We can delete the component before we compute the projection curve and add these discrete points into the topology structure after we finish the analysis. So we suppose there does not exist this kind of component. Denote $f_{0}(x, y, z)=f_{1}(x, y, z) *$ $\cdots * f_{n}(x, y, z)$. It is clear that $f(x, y, z)=0$ and $f_{0}(x, y, z)=0$ is a same surface. So we only need to determine the topology of the surface $f_{0}(x, y, z)$. Still denote it as $f(x, y, z)$. Check whether each component $f_{i}(x, y, z)$ is an opportune surface. If all components are opportune surfaces, we can compute the projection curve of $f(x, y, z)$ as follows.

For all $i, j=1,2, \cdots, n$, when $i \neq j$, denote $T_{i, j}(x, y)=\operatorname{Res}\left(f_{i}(x, y, z), f_{j}(x, y, z), z\right)$, compute its square-free polynomial which has same roots with $T_{i, j}(x, y)=0$, still denote is as $T_{i, j}(x, y)$. When $i=j$, denote the opportune projection of $f_{i}(x, y, z)$ as $T_{i, i}(x, y)$. Then the projection curve of $f(x, y, z)$ is as follows.

$$
g(x, y)=\prod_{1 \leq i \leq j \leq n} T_{i, j}(x, y) .
$$

If there exists any component which is not an opportune surface, take a transformation of coordinate system to insure that all components are opportune surfaces in the new coordinate system. And then compute the projection curve of the new surface with the method mentioned above.

For any surface $f(x, y, z)=0$, we can present the following algorithm to compute the projection curve of it which can be used to determine its topology.

Algorithm 2 The input is a polynomial $f(x, y, z)$. The output is a square-free polynomial $g(x, y)$, where $\mathcal{C}: g(x, y)=0$ is the projection curve of $f(x, y, z)$.

1. Factor $f(x, y, z)$. Suppose $f(x, y, z)=f_{1}(x, y, z)^{m_{1}} * \cdots * f_{n}(x, y, z)^{m_{n}}$, denote $f(x, y, z)=$ $f_{1}(x, y, z) * \cdots * f_{n}(x, y, z)$.

2. If $n=1$, compute the opportune projection of $\mathcal{S}$ by Algorithm 1 and return it.

3. Else $(n>1)$, do

(a) Check whether $f_{i}(x, y, z)$ is an opportune surface or not for all $i$, if there exists a component which is not an opportune surface, we should take a transformation of coordinate system as Equation (2), by the method mentioned above, such that each component is an opportune surface in the new coordinate system. Still denote the surface as $f(x, y, z)=0$.

(b) Compute the projection curve of $f(x, y, z)$ by the method discussed above. We can derive the opportune projection curve of $f(x, y, z)$ as Equation (3) and return it.

\section{Projection curve topology determination}

In this section, we will present algorithms to determine the topology of a projection curve derive from last section. 


\subsection{Preliminaries}

There are some definitions which will be used below.

A complete curve segment(CCS) of a plane algebraic curve in a finite interval is one of the following cases:

1. An isolated singularity $P_{i}$ of $\mathcal{C}: g(x, y)=0$. Denote as $C_{P_{i}}$.

2. A continuous curve segments from a singularity (or boundary point: intersection between the boundaries of a finite box and $\mathcal{C}$ ) to another singularity (or the same singularity or boundary point). Denote as $C_{i, j}^{k}$ : the k-th curve segment from the singularity $P_{i}$ to the singularity $P_{j}$; Or $C_{i, j}$ : the curve segment from the singularity $P_{i}$ to the boundary point $B_{j}$; Or $B_{i, j}^{0}$ : the curve segment form the boundary point $B_{i}$ to the boundary point $B_{j}$.

3. A closed continuous curve. Denote as $C_{Q}$, where $Q$ is a point on the closed curve.

A point $P_{0}\left(x_{0}, y_{0}\right)$ is said to be a singularity of an implicit algebraic curve $\mathcal{C}: g(x, y)=0$ if $g\left(x_{0}, y_{0}\right)=g_{x}\left(x_{0}, y_{0}\right)=g_{y}\left(x_{0}, y_{0}\right)=0$.

A critical curve of a surface is a space curve which is one part of the surface and the third parameter of the normal vector of the surface on the curve is zero.

A cell of a plane curve in a finite interval is a closed polygon whose boundaries are CCSes or boundaries of the interval.

A curve segments sequence of a singularity of a plane curve is a ordered sequence of CCSes originating from the singularity. The order is from left-up to right-up in counter-clockwise.

\section{2. topology determination}

If the opportune projection curve $\mathcal{C}: g(x, y)=0$ of $\mathcal{S}: f(x, y, z)=0$ is irreducible, then we present the following algorithm to compute the topology of $\mathcal{C}$ and obtain topology information we need in the later sections, such as singularities, CCSes, cells of the curve and so on. The plane curve topology determination algorithm already exists(Hong, 1996; Gonzalez-Vega and Necula, 2002), one can see more detail there. Our algorithm is based on the revised topology determination algorithm (Gao and Li, 2004).

Algorithm 3 Irreducible algebraic curve topology determination. The input is an irreducible plane algebraic curve $\mathcal{C}: g(x, y)=0$. The output is a bounding box $\mathcal{B}=\left\{(x, y): x_{l} \leq x \leq\right.$ $\left.x_{r}, y_{b} \leq y \leq y_{u}\right\}$, all boundary points $B_{i}\left(i \in I_{B}\right)$ defined by $\mathcal{C}$ and the boundaries of the box and four endpoints of the box, all singularities $P_{i}\left(i \in I_{S}\right)$ of $\mathcal{C}$ in the box, all CCSes of $\mathcal{C}$, such as $C_{i, j}^{k}, C_{i, l}, C_{P_{i}}, C_{Q}\left(i, j \in I_{S}, l \in I_{B}, k=0,1, \cdots\right)$ of $\mathcal{C}$ in the box and cell(s) besides it, a set of sequences of CCSes originating from each singularity, a positive number $r_{i}$ for each singularity $P_{i}$ and all cells $C_{i}\left(i \in I_{C}\right)$ in the box defined by CCSes and boundaries of the box.

1. Compute the discriminant $D(y)=\sum_{i=0}^{m} d_{i} y^{i}$ of $g(x, y)$ with respect to $x$ and let $y_{u}=$ $1+\frac{\max \left\{\left|d_{0}\right|, \cdots,\left|d_{m-1}\right|\right\}}{\left|d_{m}\right|}$ (or we can choose another rational number which is more than $\left.\frac{\max \left\{\left|d_{0}\right|, \cdots,\left|d_{m-1}\right|\right\}}{\left|d_{m}\right|}\right)$. Then by Cauchy's inequality, all the roots of $D(y)=0$ are in the interval $\left(y_{b}=-y_{u}, y_{u}\right)$. 
2. Compute the discriminant $\bar{D}(x)$ of $g(x, y)$ with respect to $y$ and determine its real roots: $\alpha_{1}<\ldots<\alpha_{s-1}$. Select two rational numbers $x_{l}$ and $x_{r}$ such that $x_{l}<\alpha_{1}$ and $x_{r}>\alpha_{s-1}$ and let $\alpha_{0}=x_{l}, \alpha_{s}=x_{r}$. Now we have determined the bounding box $\mathcal{B}$.

3. Compute the real roots of $g\left(\alpha_{0}, y\right)=0$ and $g\left(\alpha_{s}, y\right)=0$ in the interval $\left(y_{b}, y_{u}\right)$ and compute the real roots of $g\left(x, y_{b}\right)=0$ and $g\left(x, y_{u}\right)=0$ in the interval $\left(x_{l}, x_{r}\right)$. The four endpoints of the box are $\left(x_{l}, y_{u}\right),\left(x_{l}, y_{b}\right),\left(x_{r}, y_{u}\right),\left(x_{r}, y_{b}\right)$. Denote these points in order as $B_{i}, i \in I_{B}$.

4. For every $\alpha_{i}$, do

(a) compute within $\mathcal{B}$ the real roots of $g\left(\alpha_{i}, y\right), \beta_{i, 0}<\ldots<\beta_{i, t_{i}}$.

(b) For each point $P_{i, j}=\left(\alpha_{i}, \beta_{i, j}\right)$, do

i. Count the numbers of branches of $\mathcal{C}$ in $\mathcal{B}$ to the left and to the right. Denote it as $P_{l}\left(l \in I_{S}\right)$ in order if $g_{x}\left(\alpha_{i}, \beta_{i, j}\right)=g_{y}\left(\alpha_{i}, \beta_{i, j}\right)=0$, label $r_{l}=\min \left\{\alpha_{i}-\right.$ $\left.\alpha_{i-1}, \alpha_{i+1}-\alpha_{i}, \beta_{i, j}-\beta_{i, j-1}, \beta_{i, j+1}-\beta_{i, j}\right\}$ and record an ordered sequence of branches originating from the singularity from left-up to right-up in counterclockwise, transform the branches to corresponding CCSes in the end.

ii. Label each cell in $\mathbf{D}_{\mathbf{i}}=\left(\alpha_{i}, \alpha_{i+1}\right) \times\left(y_{b}, y_{u}\right)$, combine the two cells besides line segment $\overline{P_{i, j} P_{i, j+1}}$ if it is their common boundary and relabel the new cell. Denote the cells as $C_{k}\left(k \in I_{C}\right)$ in order.

iii. Label each curve segment in the interval $\mathbf{D}_{\mathbf{i}}$ and record the cell(s) besides it, combine the two curve segments(one in $\mathbf{D}_{\mathbf{i}-\mathbf{1}}$, the other in $\mathbf{D}_{\mathbf{i}}$ ) if their unique common point $P_{i, j}$ is nonsingular, relabel the new curve segment and record the cell(s) besides it. At last, we can obtain a set of CCSes and the corresponding cell(s) besides them.

5. Return corresponding information.

We can show you an example. As is shown in Fig. 1. It is a component of projection curve of the following surface.

$$
f(x, y, z)=\left(y^{2}+z^{2}-x^{2}+1 / 2 * x^{3}-4\right)^{2}-16 * x^{2}+8 * x^{3} .
$$

Its equation is $g(x, y)=2 * y^{2}-8 * y-2 * x^{2}+x^{3}+8$.

We can obtain its topology information as follows.

Boundary points set: $\left\{B_{0}, B_{1}, B_{2}, B_{3}, B_{4}, B_{5}\right\}$.

Singularities set: $\left\{P_{0}\left\{2,\left\{C_{0,0}, C_{0,3}, C_{0,0}^{0}, C_{0,0}^{0}\right\}\right\}\right\}$.

Set of CCSes: $\left\{C_{0,0}\left\{C_{0}, C_{2}\right\}, C_{0,3}\left\{C_{0}, C_{2}\right\}, C_{0,0}^{0}\left\{C_{1}, C_{2}\right\}\right\}$.

Set of cells: $\left\{C_{0}\left\{B_{0,1}, B_{1,2}, B_{2,3}, C_{0,3}, C_{0,0}\right\}, C_{1}\left\{C_{0,0}^{0}\right\}, C_{2}\left\{B_{3,4}, B_{4,5}, B_{5,0}\right.\right.$, $\left.\left.C_{0,0}, C_{0,0}^{0}, C_{0,3}\right\}\right\}$.

When $g(x, y)$ is reducible, we can compute a finite box and the real roots of the discriminant of each component of $g(x, y)$ with respect to $y$ as Algorithm 3. Replace all these roots in order, then compute the topology information of $g(x, y)=0$ as Algorithm 3. We can derive the topology of $g(x, y)=0$ in the end. 


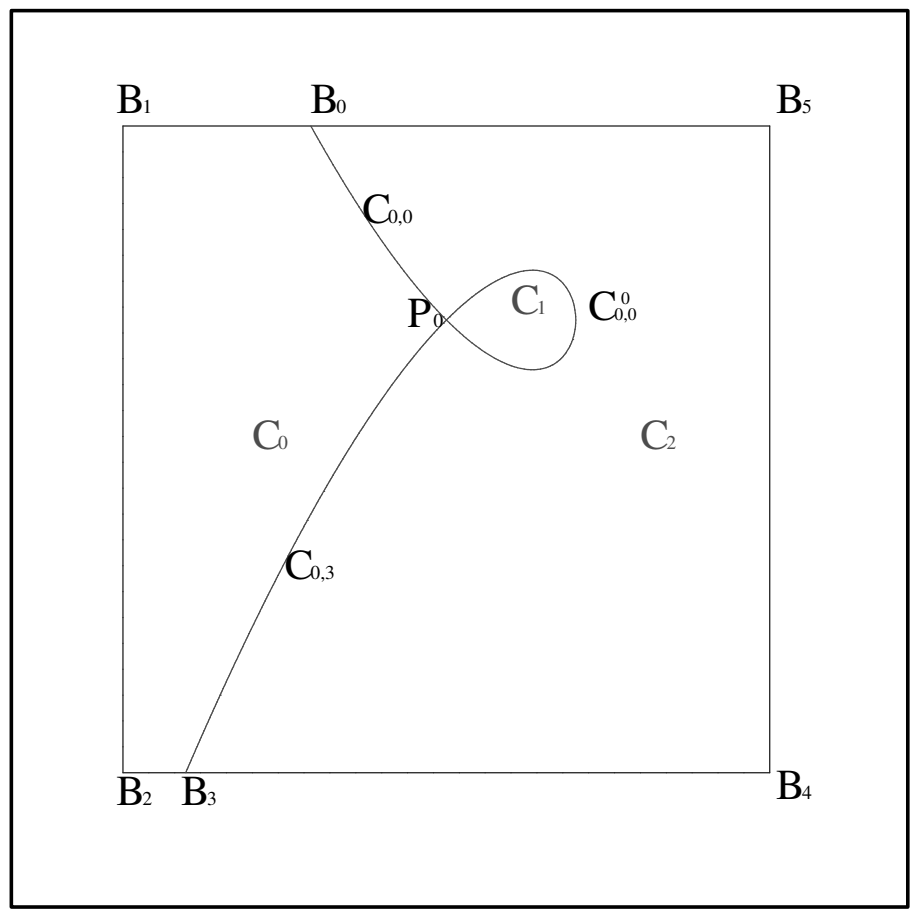

Fig. 1. Topology determination of irreducible plane curve

The following algorithm is to determine the topology of any square-free algebraic curve $g(x, y)=0$. In order to fit our problem and describe easily, we can suppose $g(x, y)=$ $g_{1}(x, y) \cdots g_{m}(x, y)$ if it is reducible.

Algorithm 4 Plane curve topology determination. The input is $\mathcal{C}: g(x, y)=0$. The output is same to Algorithm 3.

1. If $g(x, y)$ is irreducible, determine the topology of $\mathcal{C}$ by Algorithm 3.

2. Else $(g(x, y)$ is reducible), do as follows.

(a) For each $g_{i}(x, y)$, compute a finite box with the method presented in Algorithm 3. Search a new finite box which includes all the finite boxes above as the finite box of $g(x, y)=0$. Compute the intersection points of the box boundary and $g(x, y)=0$.

(b) Separate the vertical line(s) $L_{t}(x, y)=x-c_{t}=0(t=0, \cdots, L)$ from $g(x, y)$ if it exists. Denote all the left components of $g(x, y)$ as $g_{0}(x, y)$. Suppose it as $g_{0}(x, y)=g_{1}(x, y) \cdots g_{m_{1}}(x, y)$.

(c) Solve $\operatorname{Res}\left(g_{i}(x, y), \frac{\partial g_{i}(x, y)}{\partial y}, y\right)=0$ and $\operatorname{Res}\left(g_{i}(x, y), g_{j}(x, y), y\right)=0$ for all $i, j=$ $0, \cdots, m_{i}(i \neq j)$. Denote their roots and $c_{t}(t=0, \cdots, L)$ together in order as $\alpha_{k}\left(k=0, \cdots, l, \alpha_{0}=x_{l}, \alpha_{l}=x_{r}, \alpha_{k}<\alpha_{k+1}\right)$.

(d) For every $\alpha_{k}$, to $g_{0}(x, y)$, we can do the same work as Algorithm 3 in step 4. Note that when $\alpha_{k}=c_{t}(t=0, \cdots, L)$, all the real roots of $g_{0}\left(\alpha_{k}, y\right)=0$, denote as $\beta_{k, j}\left(j=0, \cdots, t_{k}\right)$, are singularities of $g(x, y)=0$, and line segments 
$\overline{P_{k, j} P_{k, j+1}}\left(k=0, \cdots, t_{k}-1\right), \overline{B_{k_{1}} P_{k, 0}}, \overline{P_{k, t_{k}} B_{k_{2}}}$ are CCSes of $g(x, y)=0$. We can derive the topology information of $g(x, y)=0$ in the end.

3. Return the corresponding topological information of $\mathcal{C}$.

Here is an example that $g(x, y)$ is reducible. It is the projection curve of surface defined by Equation (4). Its equation is as follows.

$$
g(x, y)=g_{1}(x, y) * g_{2}(x, y) * g_{3}(x, y) * g_{4}(x, y),
$$

where $g_{1}(x, y)=x, g_{2}(x, y)=(x-2), g_{3}(x, y)=2 * y^{2}+8 * y-2 * x^{2}+x^{3}+8, g_{4}(x, y)=$ $2 * y^{2}-8 * y-2 * x^{2}+x^{3}+8$. We can derive its picture as Fig. 2 .

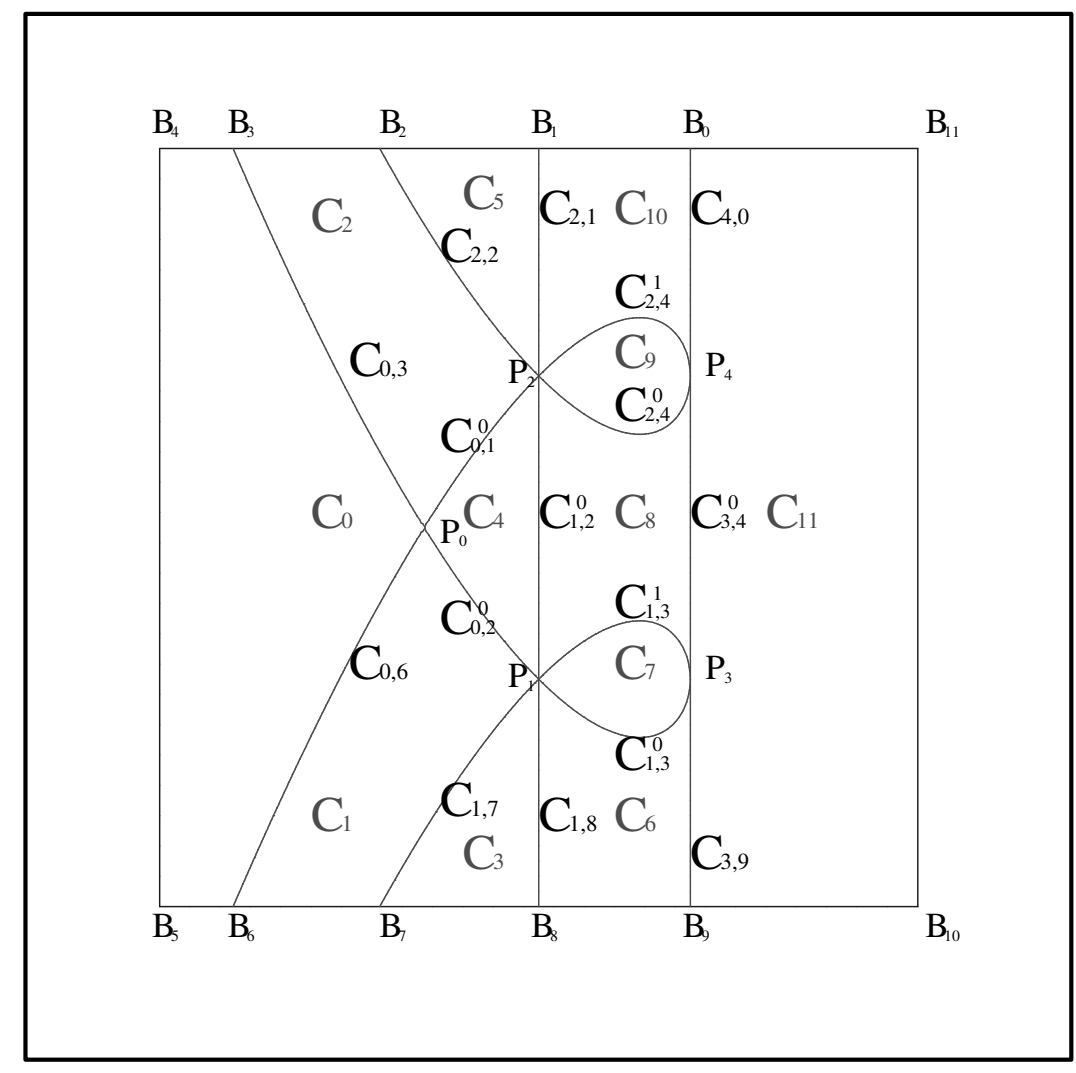

Fig. 2. Topology determination of reducible plane curve

\section{Space curve segmentation and surface patch composition}

In this section, we will determine the position of each space curve segment and each surface patch of $\mathcal{S}$. Our basic idea to determine the topology of $\mathcal{S}$ is to divide $\mathcal{S}$ into points, space curve segments and surface patches, from points to obtain space curve segments, form space curve segments to obtain surface patches, at last to obtain the topology of the surface.

The following is our main steps. 
At first, we need to determine the points of $\mathcal{S}$ on each line lifting from a boundary point $B_{i}\left(\bar{x}_{i}, \bar{y}_{i}\right)\left(i \in I_{B}\right)$ or a singularity $P_{i}\left(x_{i}, y_{i}\right)\left(i \in I_{S}\right)$ of $\mathcal{C}: g(x, y)=0$, especially from a singularity of $\mathcal{C}$. These points are the endpoints of CSCSes. Second, we need to determine how many CSCSes originating from each point of a certain line on each CCP whose boundary is the line. Then we can determine all CSCSes of $\mathcal{S}$. Third, we need to compute the number of CSPs originating from each CSCS in the corresponding cell body(s). At last, we can determine the CSPs in each cell body from below to up by pointing out their boundaries, those are CSCSes. So, we determine each CSP of $\mathcal{S}$. It is what we want.

\subsection{Basic definition}

In order to describe our algorithm clearer, we present the following definitions.

A complete cylinder patch (CCP) is a cylinder patch lifting from a CCS obtained in last section.

A cell body is a body lifting from a cell obtained in last section. There are two cell bodys beside a CCP corresponding to the cells beside the CCS. When the CCS is a point, there is only one cell body beside it.

A complete space curve segment (CSCS) of $\mathcal{S}: f(x, y, z)=0$ is one of space curve segments defined by a CCP and $\mathcal{S}$.

A complete surface patch (CSP) of $\mathcal{S}: f(x, y, z)=0$ is a surface patch which is part of $\mathcal{S}$, and its boundary is CSCSes. by

Given a function $P(x)$, let $P_{0}(x)=P(x), P_{1}(x)=P^{\prime}(x)$ and define the Sturm functions

$$
P_{i}(x)=-\left(P_{i-2}(x)-P_{i-1}(x)\left[\frac{P_{i-2}(x)}{P_{i-1}(x)}\right]\right),
$$

where $\left[\frac{P_{i-2}(x)}{P_{i-1}(x)}\right]$ is a polynomial quotient. The chain is terminated when $P_{n}(x)$ is a constant. Then $P_{0}(x), P_{1}(x), \cdots, P_{n}(x)$ is the Sturm functions of $P(x)$.

Sign-changing number of Sturm functions of $P(x)$ at point $x=a$ is the number of sign changes on the Sturm functions of $P(x)$ evaluated at point $x=a$. That is, the number of sign changes on $P_{0}(a), P_{1}(a), \cdots, P_{n}(a)$.

\subsection{Basic algorithm}

To do so, we need the following basic algorithms.

The following algorithm is to isolate the real roots of an equation $T(x)$. The difference between the algorithm and general algorithm is that the isolated points of our algorithm is not a root of $T(x)$.

Algorithm 5(Real Roots-Isolating) The input is Sturm functions of polynomial $T(x)$ and an interval $(a, b)(T(a) \neq 0, T(b) \neq 0)$. The output is a serial of ordered rational number in $(a, b)$, there is a real root between each pair of adjacent numbers.

1. Compute the sign-changing numbers $V(a), V(b)$ of the Sturm functions of $T(x)$ at $x=a, x=b$ respectively. $V(a)-V(b)$ is the number of real roots between $(a, b)$ by Sturm theorem. Let rational numbers set be $N_{s}:=a, b$. If $V(a)-V(b)=0$, return $\emptyset$. If $V(a)-V(b)=1$, return $N_{s}$.

2. When $V(a)-V(b)>1$, if $T\left(\frac{a+b}{2}\right) \neq 0, c=\frac{a+b}{2}$, else choose another rational number $c$ near $\frac{a+b}{2}$ in $(a, b)$ insuring that $T(c) \neq 0$. 
(a) If $V(a)-V(c)>1$ and $V(c)-V(b)>1, N_{s}:=N_{s}+\{c\}$, do $a=a, b=c$, go to 2 ; and $a=c, b=b$, go to 2 .

(b) Else if $V(a)-V(c)=1$ and $V(c)-V(b)>1, N_{s}:=N_{s}+\{c\}$, and let $a=c, b=b$, go to 2 .

(c) Else if $V(a)-V(c)>1$ and $V(c)-V(b)=1, N_{s}:=N_{s}+\{c\}$, and let $a=a, b=c$, go to 2 .

(d) Else if $V(a)-V(c)=0$ and $V(c)-V(b)>1$, let $a=c, b=b$, go to 2 .

(e) Else if $V(a)-V(c)>1$ and $V(c)-V(b)=0$, let $a=a, b=c$, go to 2 .

3. Return the ordered rational numbers $N_{s}$.

Given a point, a positive number and a plane curve (the point can be on the curve or not on the curve), the following algorithm is to find the circle whose center is the point, and it is the minimal circle among the circles which is tangent to the curve. Return a positive number which is equal to half of the less of the radius and the given positive number.

Algorithm 6 The input is a plane algebraic curve $T(x, y)=0$, a positive number $r$ and a point $P_{0}\left(x_{0}, y_{0}\right)$. The output is a positive number which is equal to half of the minimal of extremum distance $r_{\text {min }}$ from $P_{0}$ to the curve and $r$.

1. Let $L(x, y, \lambda)=\left(x-x_{0}\right)^{2}+\left(y-y_{0}\right)^{2}+\lambda T(x, y)$.

2. Eliminate $\left\{2\left(x-x_{0}\right)+\lambda T_{x}(x, y), 2\left(y-y_{0}\right)+\lambda T_{y}(x, y), T(x, y)\right\}$ in order $\{\lambda \succ x \succ y\}$.

3. Solve the univariate equation in $y$ obtaining from last step in interval $\left(y_{0}-r, y_{0}+r\right)$. If there is no real root in the interval, return $\mathrm{r}$; Else, get corresponding $x_{i, j}$ for each real root $y_{i}$ we have gotten in the interval $\left(x_{0}-r, x_{0}+r\right)$, if there is no real root in the interval, return $r / 2$; else, let $R=\min _{i, j} \sqrt{\left(x-x_{i, j}\right)^{2}+\left(y_{0}-y_{i}\right)}$, if $R \leq r$, return $R / 2$, else, return $r / 2$.

Remark The step 2 of this algorithm is an application of Wu's method. One can find more detail in [14].

\subsection{Algorithm of segmentation}

To each singularity $P_{i}\left(i \in I_{S}\right)$ of $g(x, y)=0$, there is a sequence of CCSes $C_{i, j_{1}}^{k_{1}}, \cdots, C_{i, j_{t}}^{k_{t}}$. Lifting them up, we can obtain a sequence of $\operatorname{CCPs} S C_{i, j_{1}}^{k_{1}}, \cdots, S C_{i, j_{t}}^{k_{t}}$. The singularity corresponds to a vertical line $\left\{x=x_{P_{i}}, y=y_{P_{i}}\right\}$. There are some points $V_{i, j}\left(j=0, \cdots, s_{i}\right)$ of $\mathcal{S}$ on the line and some CSCSes $C_{i, j_{l}}^{k_{l}, m}\left(m=0, \cdots, t_{i, j, k}\right)$ on each CCP $S C_{i, j_{l}}^{k_{l}}(l=1,2, \cdots, t)$. we need to determine the CSCSes originating from $V_{i, j}$ on each CCP. The following algorithm is to finish the task.

Algorithm 7 The input is a real algebraic curve $\mathcal{C}: g(x, y)=0$, a real algebraic surface $\mathcal{S}: f(x, y, z)=0$, a singularity $P_{i}\left(x_{i}, y_{i}\right)$ on $\mathcal{C}$, a positive number $r_{i}$ corresponding to $P_{i}$ and a sequence of CCSes $\left\{C_{i, j_{1}}^{k_{1}}, \cdots, C_{i, j_{t}}^{k_{t}}\right\}$ obtained in Algorithm 4. The output is a sequence of points of $\mathcal{S}$ on the line lifting from $P_{i}$, a set of sequences of CSCSes $\left\{C_{i, j_{l}}^{k_{l}, m}, m=0, \cdots, t_{i, j, k}\right\}$ for each $C_{i, j_{l}}^{k_{l}}$. Note that we only know one endpoint of the CSCSes, the other is unknown. 
1. Isolate the real roots of $f\left(x_{i}, y_{i}, z\right)=0$ by Algorithm 5 and obtain the isolating values $z_{i, 0}, z_{i, 1}, \cdots, z_{i, s_{i}}$. Denote $\left(x_{i}, y_{i}, z_{i, j}\right)$ as $W_{i, j}$. There exists a point of $\mathcal{S}, V_{i, j}$, which is on the line $\left\{x=x_{i}, y=y_{i}\right\}$ between points $W_{i, j}$ and $W_{i, j+1}$.

2. For $r_{i}, P_{i}, g(x, y)=0$, we can obtain a positive number $R_{i}$ by Algorithm 6 . It clear that the number of intersection points of the circle $\left(x-x_{i}\right)^{2}+\left(y-y_{i}\right)^{2}=r^{2}\left(0<r \leq R_{i}\right)$ and $\mathcal{C}$ is equal to the number of the CCSes in the input sequence.

3. In plane $z=z_{i, j}\left(j=0,1, \cdots, s_{i}\right)$, for $R_{i}, P_{i}, f\left(x, y, z_{i, j}\right)=0$ we can obtain a positive number $r_{i, j}$ by Algorithm 6. Still denote the minimal among $\left\{r_{i, 0}, \cdots, r_{i, s_{i}}\right\}$ as $r_{i}\left(r_{i} \leq\right.$ $\left.R_{i}\right)$.

4. Compute the real intersection points of the equations $\left\{\left(x-x_{i}\right)^{2}+\left(y-y_{i}\right)^{2}=r_{i}^{2}, g(x, y)=\right.$ $0\}$. We can determine each point on its corresponding CCS by its coordinate and the order of CCSes in the sequence. Denote them as $\left\{P_{i, j_{1}}, P_{i, j_{2}}, \cdots, P_{i, j_{t}}\right\}$.

5. For each $P_{i, j_{l}}\left(x_{i, j_{l}}, y_{i, j_{l}}\right)(l=1, \cdots, t)$, compute the number of real roots of $f\left(x_{i, j_{l}}, y_{i, j_{l}}, z\right)=$ 0 in interval $\left(z_{i, j}, z_{i, j+1}\right)\left(j=0,1, \cdots, s_{i}-1\right)$. It is the number of CSCSes originating from $V_{i, j}$ on the CCP $S C_{i, j_{l}}^{k_{l}}$. So we can determine the CSCSes on each CCP: one of their two endpoints is on the line lifting from $P_{i}$, their order on the CCPs is from below to top. Denote them as $C_{i, j_{l}}^{k_{l}, m}$. If there does not exist real root in the interval $\left(z_{i, 0}, z_{i, s_{i}}\right)$, delete the CCS from the input sequence of CCSes.

6. Return the corresponding information.

Remark In Algorithm 7, if the singularity is a isolated point, we need not to compute it by this algorithm. The input sequence of CCSes may includes CCS like $C_{i, j}$ (the endpoints are a singularity and a boundary point). It is also OK for the Algorithm. And the CSCSes on the CCP lifting from $C_{i, j}$ are determined by computing $P_{i}$ with this algorithm.

We also need to compute the CSCSes on the CCPs lifting from boundary line segments, for example, $B_{i, i+1}$ or CCS $B_{i, j}^{0}$. It is easy. We can revise Algorithm 7 to do so. Isolate $f\left(x_{B_{i}}, y_{B_{i}}, z\right)=0$, compute line segments replacing the discs in Algorithm 7. The line segments are parallel to XY-plane and their centers are projected to $B_{i}$. The following algorithm is to an algorithm revised from Algorithm 7 to compute corresponding information for a boundary point. Note that the number of CSCSes on the CCP $S C_{j, i}$ is the number of real roots of $f\left(x_{B_{i}}, y_{B_{i}}, z\right)=0$. The CSCSes on CCP $S B_{i, i+1}$ is unknown, because $C_{j, i}$ may be the projection of a singular curve of $f(x, y, z)=0$.

Algorithm 8 The input is a real algebraic curve $\mathcal{C}: g(x, y)=0$, a real algebraic surface $\mathcal{S}: f(x, y, z)=0$, a boundary point $B_{i}\left(x_{i}, y_{i}\right)\left(i \in I_{B}\right)$, a $\operatorname{CCS} C_{j, i}\left(j \in I_{S}\right)$ obtained in Algorithm 4. The output is an empty set or a sequence of points of $\mathcal{S}$ on the line lifting from $B_{i}$ (if they exist), sequences of CSCSes on the CCPs lifting from the CCS $C_{j, i}$ and boundary line segments $B_{i, i+1}, B_{i, i-1}$. Denote as $C_{j, i, m}, B_{i, i+1}^{m_{1}}, B_{i, i-1}^{m_{2}}$. Note that we only know one endpoint of the CSCSes, the other is unknown.

1. Isolate the real roots of $f\left(x_{i}, y_{i}, z\right)=0$ by Algorithm 5 .

(a) If there is no real root, delete the $\operatorname{CCS} C_{j, i}$, combine the two cells divided by it and return an empty set. 
(b) Else, do the following.

i. We can obtain the isolating values $z_{i, 0}, z_{i, 1}, \cdots, z_{i, s_{i}}$. Denote $\left(x_{i}, y_{i}, z_{i, j}\right)$ as $W_{i, j}^{0}$. There exists a point of $\mathcal{S}, V_{i, j}^{0}$, which is on the line $\left\{x=x_{i}, y=y_{i}\right\}$ between points $W_{i, j}^{0}$ and $W_{i, j+1}^{0}$.

ii. Denote the CSCSes on the CCP lifting from $C_{j, i}$ as $C_{i, j, m}$ in order.

iii. Parameterize the boundary line of the finite box as $\left(t a+x_{i}, t b+y_{i}\right)$.

iv. Compute the real roots of the equation $f\left(t a+x_{i}, t b+y_{i}, z_{j}\right)=0$ for each $j=0,1, \cdots, s_{i}$. Record the root whose absolute value is the minimal among the real root(s). Denote the absolute value of the root as $r_{j}$. And denote the half of the minimal among $r, r_{0}, r_{1}, \cdots, r_{s_{i}}$ as $R$.

v. Compute the number of real roots of $f\left(R a+x_{i}, R b+y_{i}, z\right)=0$ and $f(-R a+$ $\left.x_{0},-R b+y_{0}, z\right)=0$ in the interval $\left(z_{m}, z_{m+1}\right)\left(m=0, \cdots, s_{i}-1\right)$ respectively. They are the numbers of CSCSes originating from $V_{i, m}^{0}$ on the CCPs beside the line lifting from $B_{i}$. Denote the CSCSes as $B_{i, i+1, m_{1}}$ and $B_{i, i-1, m_{2}}$ in order.

2. Return the corresponding information.

Remark To one of the four endpoints of the box $B_{i}$, if it is not a point of $\mathcal{C}$, the number of the points on the line lifting form it (if it exists) is equal to the number of CCSes on the CCP $S B_{i, i+1}$ or $S B_{i, i-1}$. So we need not to compute these points.

After computing all boundary points and singularities of $\mathcal{C}$ by Algorithm 7 or Algorithm 8, we can determine the position of all CSCSes of $\mathcal{S}$.

And now, we need to compute the number of CSPs originating from each CSCS in the two cell bodies beside the CCP which the CSCS lies on. To do so, we need to compute two points in the cells beside the corresponding CCS $C_{i, j}^{k}$ (or $C_{i, j}$ ) on each side of the point $P_{i, j}^{k}$ or $P_{j, i}^{k}$ ( or $P_{i, j}$ ) obtained in Algorithm 7. Here we only need one point on the CCS. Lift the point up and isolate the real roots of $\mathcal{S}$ on the line: $z_{0}, z_{1}, \cdots, z_{l_{i, j, k}}$. Compute a sequence of line segments whose center are the isolated points and all have a same direction and are parallel to XY-plane. Insure that all line segments have no common point with $\mathcal{S}$. Suppose the shortest length is $r_{i, j, k}\left(r_{i, j}\right)$. Choose the two endpoints $P_{i, j}^{k, 0}, P_{i, j}^{k, 1}$ or $P_{i, j, 0}, P_{i, j, 1}$ of the line segment on the plane where $\mathcal{C}$ lies on. Lift the points up, compute the number of real roots of $\mathcal{S}$ on each line in the interval $\left(z_{s}, z_{s+1}\right)\left(s=0, \cdots, l_{i, j, k}\right)$. It is the number of CSPs originating from $\operatorname{CSCS} C_{i, j}^{k, s}$ in a cell body. The following algorithm is to do the work.

Algorithm 9 The input is a real algebraic curve $\mathcal{C}: g(x, y)=0$, a real algebraic surface $\mathcal{S}: f(x, y, z)=0$, a CCS $C_{i, j}^{k}\left(\right.$ or $\left.C_{i, j}\right)$ on $\mathcal{C}$ and two cells beside it, a points on the CCS: $P_{i, j}^{k}\left(\right.$ or $\left.P_{i, j}\right)\left(x_{0}, y_{0}\right)$ and a sequence of CSCSes $\left\{C_{i, j}^{k, m}, m=0, \cdots, l_{i, j, k}-1\right\}$ on the CCP lifting from the CCS. The output is the number of CSPs originating from each CSCS of the sequence in the two cell bodies respectively.

1. Compute the tangent line of $\mathcal{C}$ at point $P_{i, j}^{k}$, compute the vertical line of the line at $P_{i, j}^{k}$ and parameterize it as $\left(t a+x_{0}, t b+y_{0}\right)$.

2. Compute the real roots of the equation $g\left(t a+x_{0}, t b+y_{0}\right)=0$. Record the root whose absolute value is the minimal among the nonzero real root(s). If the root does not exist, denote $r$ as a constant, such as 1, else denote $r$ as the absolute value of the root. 
3. Isolate the real roots of $f\left(x_{0}, y_{0}, z\right)=0$ by Algorithm 5 , and derive a sequence of rational number $\left\{z_{0}, z_{1}, \cdots, z_{l_{i, j, k}}\right\}$.

4. Compute the real roots of the equation $f\left(t a+x_{0}, t b+y_{0}, z_{i}\right)=0$ for each $i=$ $0,1, \cdots, l_{i, j, k}$. Record the root whose absolute value is the minimal among the real $\operatorname{root}(\mathrm{s})$. Denote the absolute value of the root as $r_{i}$. And denote the half of the minimal among $r, r_{0}, r_{1}, \cdots, r_{l_{i, j, k}}$ as $R$.

5. Compute the number of real roots of $f\left(R a+x_{0}, R b+y_{0}, z\right)=0$ and $f\left(-R a+x_{0},-R b+\right.$ $\left.y_{0}, z\right)=0$ in the interval $\left(z_{m}, z_{m+1}\right)\left(m=0, \cdots, l_{i, j, k}-1\right)$ respectively. They are the numbers of CSPs in the cell bodies beside the CCP $S C_{i, j}^{k}$.

6. Return the corresponding information.

Remark If the CCS is an isolated singularity of $\mathcal{C}$, we only need to lift the point up, isolate the real roots of $S$ on the line by Algorithm 5, find a line segments (its direction is parallel to XY-plane) which pass the point as Algorithm 9, then we can easily determine the number of CSPs originating from the points of $S$ on the lifting line. If the CCS is a closed curve, $Q$ is a point on the CCS, we can also easily compute the number of CSPs originating from the CSCSes of the CCP lifting from the CCS like the Algorithm 9.

And now, we have determine the position of all CSCSes and the number of CSPs originating from each CSCS in the two cell bodies beside it. Then we can form CSPs of $\mathcal{S}$.

For each cell body lifting from the cell of $\mathcal{C}$, because the number of CSPs originating from the CSCSes on each CCPs of the cell body is the same, we can determine each CSP by point out its boundaries, CSCSes.

The following algorithm is to determine the CSPs of $\mathcal{S}$ by the topology information obtained by Algorithm 4 and $\mathcal{S}$.

Algorithm 10 The input is $\mathcal{S}: f(x, y, z)=0$ and the output of Algorithm 4. The output is all the CSPs of $\mathcal{S}$.

1. Compute all the singularity of $\mathcal{C}$ by Algorithm 7 and all boundary points of $\mathcal{C}$ by Algorithm 8, determine all the CSCSes on each CCP lifting from the CCS of $\mathcal{C}$.

2. Compute the number of CSPs originating from each CSCS in two cell bodies beside it by Algorithm 9.

3. For each cell body lifting from the cell of $\mathcal{C}$, because the number of CSPs originating from the CSCSes on each CCPs of the cell body is the same, we can determine each CSP by point out its boundaries-CSCSes.

4. Return the CSPs of $\mathcal{S}$.

And now, we have gotten the CSPs of $S$, it is clear that has a same topology with all the CSPs we obtained by Algorithm 10.

Here is an example. The given surface is a torus. Its equation is as follows.

$f(x, y, z)=2 * y^{2} * x^{2}+8 * y^{2}-17 * z^{2}+y^{4}+x^{4}+2 * x^{2} * z^{2}+2 * y^{2} * z^{2}+z^{4}+16-17 * x^{2}$. 


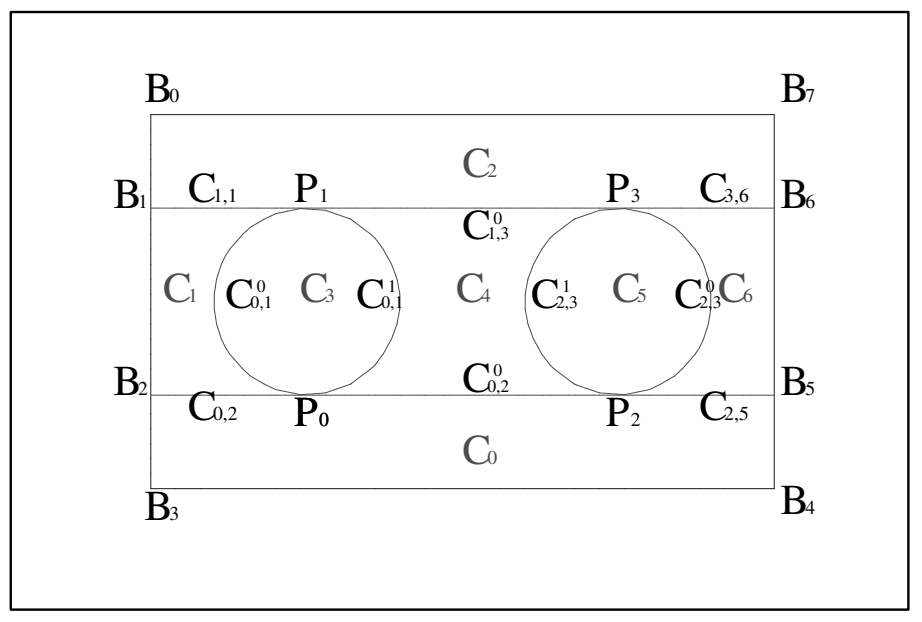

Fig. 3. Topology determination of a projection curve of a torus

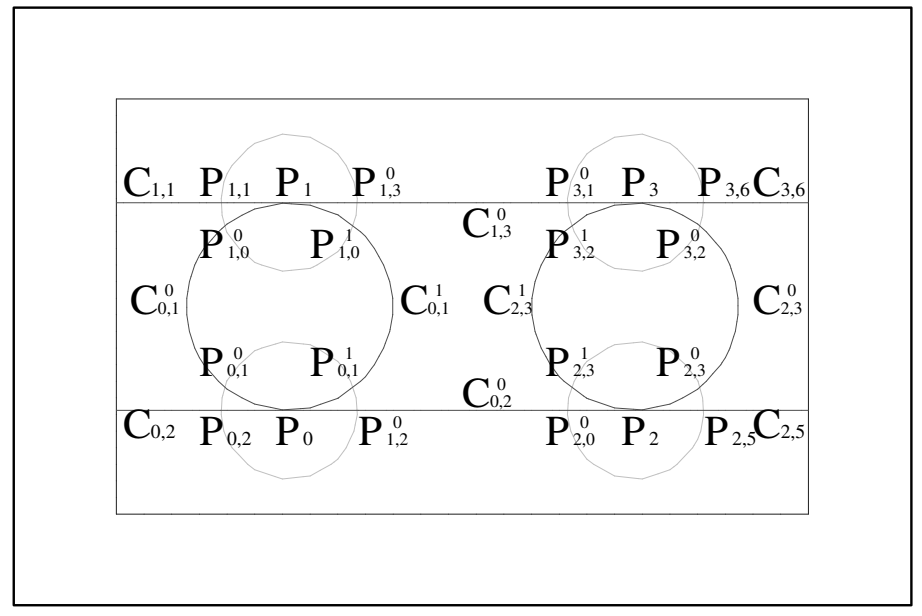

Fig. 4. Topology determination of a projection curve of a torus

Its projection curve is as follows obtained by Algorithm 2. And its topology information is as Fig. 3.

Follow Algorithm 10, isolate four singularities we can know that there is only one point on each line lifting from the singularities. Denote them as $V_{0,0}, V_{1,0}, V_{2,0}, V_{3,0}$. The radii of circles whose centers are the singularities are all 1 . We can get four points beside each singularities, as is shown in Fig. 4. Isolate the real roots of the torus on the lines lifting from the four points, for example, the four points of singularity $P_{1}: P_{1,1}, P_{1,0}^{0}, P_{1,0}^{1}, P_{1,3}^{0}$. We can find that there is no real root of the torus on the line lifting from $P_{1,1}$, so delete the CCS $C_{1,1}$ and combine its corresponding cells $C_{1}, C_{2}$. There are one, two and three real roots on the lines lifting from $P_{1,0}^{0}, P_{1,0}^{1}, P_{1,3}^{0}$ respectively. So as other singularities. Then we know that there are one CSCS on CCPs $S C_{0,1}^{0}, S C_{2,3}^{0}$, denote as $C_{0,1}^{0,0}, C_{2,3}^{0,0}$, two CSCSes on CCPs $S C_{1,3}^{0}, S C_{0,2}^{0}$, denote as $C_{1,3}^{0,0}, C_{1,3}^{0,1}, C_{0,2}^{0,0}, C_{0,2}^{0,1}$, and three CSCSes on CCPs $S C_{0,1}^{1}, S C_{2,3}^{1}$, denote 
as $C_{0,1}^{1,0}, C_{0,1}^{1,1}, C_{0,1}^{1,2}, C_{2,3}^{1,0}, C_{2,3}^{1,1}, C_{2,3}^{1,2}$. In order to compute the number of CSPs originating from each CSCS, we will compute two points $P_{i, j}^{k, 0}, P_{i, j}^{k, 1}$ beside $P_{i, j}^{k}$ in two cells by Algorithm 9. As is shown in Fig. 5. $P_{1,3}^{0,0}, P_{1,3}^{0,1}$ are two points beside $P_{1,3}^{0}$ in cells $C_{0}, C_{4}$ respectively. Lift them up, we can find there is no real root of the torus on the line lifting form $P_{1,3}^{0,0}$ and there are two CSPs originating from CSCSes $C_{1,3}^{0,0}, C_{1,3}^{0,1}$ in $C_{4}$ respectively. In the same way, we can derive the number of CSPs originating from each CSCS in each cell beside it. So we can get the CSPs as follows.

The CSPs lifting from $C_{3}$ :

$$
\left\{S_{3}^{0}:\left[C_{0,1}^{0,0}, C_{0,1}^{1,0}\right], S_{3}^{1}:\left[C_{0,1}^{0,0}, C_{0,1}^{1,2}\right]\right\} .
$$

The CSPs lifting form $C_{4}$ :

$$
\begin{aligned}
& \left\{S_{4}^{0}:\left[C_{0,1}^{1,0}, C_{0,2}^{0,0}, C_{2,3}^{1,0}, C_{1,3}^{0,0}\right], S_{4}^{1}:\left[C_{0,1}^{1,1}, C_{0,2}^{0,0}, C_{2,3}^{1,1}, C_{1,3}^{0,0}\right]\right. \\
& \left.S_{4}^{2}:\left[C_{0,1}^{1,1}, C_{0,2}^{0,1}, C_{2,3}^{1,1}, C_{1,3}^{0,1}\right], S_{4}^{3}:\left[C_{0,1}^{1,2}, C_{0,2}^{0,1}, C_{2,3}^{1,2}, C_{1,3}^{0,1}\right]\right\}
\end{aligned}
$$

The CSPs lifting from $C_{5}$ :

$$
\left\{S_{5}^{0}:\left[C_{2,3}^{1,0}, C_{2,3}^{0,0}\right], S_{5}^{1}:\left[C_{2,3}^{1,2}, C_{2,3}^{0,0}\right]\right\} .
$$

There is no CSP lifting from $C_{0}$.

Then we get what we want.

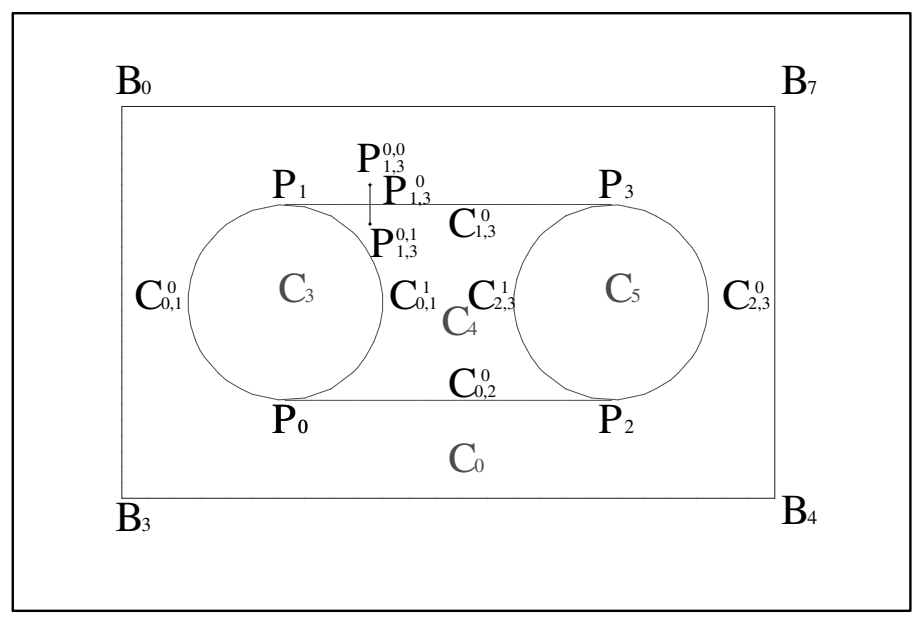

Fig. 5. Topology determination of a projection curve of a torus

\section{Combination of surface patches and surface topology representation}

As we known, there are many CSCSes of $\mathcal{S}$ which are not critical curve segments. In order to simplify the representation of the topology of $\mathcal{S}$ without changing it, we can combine any two CSPs who have a common CSCS which is not a critical curve segment. 
For each CCP which is not lifted from a CCS which is a boundary line segment of $C$, check each CSCS on the CCP whether the numbers of CSPs originating from it in the two cell bodies both are 1. If it is, combine the two CSPs in the two cell bodies as follows.

$$
\begin{gathered}
S_{i_{1}}^{j_{1}}=\left[\cdots, C_{i^{\prime}, i}^{k_{1}, m_{1}}, C_{i, j}^{k, m}, C_{j, j^{\prime}}^{k_{2}, m_{2}}, \cdots\right] \\
S_{i_{2}}^{j_{2}}=\left[\cdots, C_{i^{\prime \prime}, i}^{k_{3}, m_{3}}, C_{i, j}^{k, m}, C_{j, j^{\prime \prime}}^{k_{4}, m_{4}}, \cdots\right] \\
S_{i_{1}}^{j_{1}}+S_{i_{2}}^{j_{2}}=\left[\cdots, C_{i^{\prime}, i}^{k_{1}, m_{1}}, C_{i, i^{\prime \prime}}^{k_{3}, m_{3}}, \cdots, C_{j^{\prime \prime}, j}^{k_{4}, m_{4}}, C_{j, j^{\prime}}^{k_{2}, m_{2}}, \cdots\right]=S_{i_{1}, i_{2}}^{j_{1}, j_{2}},
\end{gathered}
$$

where $S_{i_{1}}^{j_{1}}, S_{i_{2}}^{j_{2}}$ are the $j_{1}$-th CSP in the cell body $C B_{i_{1}}$ and the $j_{2}$-th CSP in the cell body $C B_{i_{2}}$. They are presented by their boundaries-CSCSes as Equations (5) and (6). $C B_{i_{1}}, C B_{i_{2}}$ have a common boundary: $\operatorname{CCP} C_{i, j}^{k}$. And there is only one CSP originating from CSCS $C_{i, j}^{k, m}$ in each cell body $C B_{i_{1}}, C B_{i_{2}}$. Then we can combine the CSPs as above.

In the end, we can get a set of surface patches whose boundary curve segments are critical curve segments.

The following is an algorithm to combine the common CSCS, where is only one CSP orginating from it in the each cell body beside it, of two CSPs in the two cell bodies beside it.

Algorithm 11 The input is the output of Algorithm 10. The output is a simplified set of CSPs of $\mathcal{S}$. And the boundaries of the CSPs are critical curve segments of $\mathcal{S}$.

1. For any two adjacent cell bodies and their common CCP, from the below to the up, find out the CSCSes, where there is only one CSP originating from them in each cell body beside them, combine the two CSPs as formula (7).

2. Return the CSPs of the $\mathcal{S}$ until the surface patches of it can not be combined.

We will follow the algorithm to finish the example in last section. Because there is no CSPs in $C_{0}$, we only need to consider the CSCSes on CCPs $S C_{0,1}^{1}, S C_{2,3}^{1}$. There is only one CSP originating from $C_{0,1}^{0,0}$ in $C_{3}, C_{4}$ respectively. So we can combine the CSPs $S_{3}^{0}, S_{4}^{0}$ as follows.

$$
S_{3,4}^{0,0}=S_{3}^{0}+S_{4}^{0}=\left[C_{0,1}^{0,0}, C_{0,2}^{0,0}, C_{2,3}^{1,0}, C_{1,3}^{0,0}\right]
$$

At last, we can obtain four surface patches of the torus as follows, whose boundary space curve segments are critical curve of the torus.

$$
\begin{gathered}
S_{3,4,5}^{0,0,0}=\left[C_{0,1}^{0,0}, C_{0,2}^{0,0}, C_{2,3}^{0,0}, C_{1,3}^{0,0}\right], \\
S_{3,4,5}^{1,3,1}=\left[C_{0,1}^{0,0}, C_{0,2}^{0,1}, C_{2,3}^{0,0}, C_{1,3}^{0,1}\right], \\
S_{4}^{1}=\left[C_{0,1}^{1,1}, C_{0,2}^{0,0}, C_{2,3}^{1,1}, C_{1,3}^{0,0}\right], \\
S_{4}^{2}=\left[C_{0,1}^{1,1}, C_{0,2}^{0,1}, C_{2,3}^{1,1}, C_{1,3}^{0,1}\right] .
\end{gathered}
$$




\section{Main Algorithm}

By the discussion in the former sections, we can present the main algorithm to determine the topology of an implicit algebraic surface.

Algorithm 12 The input is an implicit algebraic surface $\mathcal{S}: f(x, y, z)=0$. The output is a set of surface patches whose boundaries are critical curve segments.

1. Get the projection curve $\mathcal{C}: g(x, y)=0$ of $\mathcal{S}$ by Algorithm 2 .

2. Topology determination of $\mathcal{C}$ by Algorithm 4 .

3. Space curve segmentation and surface patch segmentation of $\mathcal{S}$ by Algorithm 10 .

4. Combination of CSPs of $\mathcal{S}$ by Algorithm 11.

5. Return a set of surface patches which are critical curve segments of $\mathcal{S}$.

One can find our algorithm is effective in determining the topology of algebraic surface by the examples shown above.

It is difficult to analyze the computing time of the algorithm. The more the singularities of $\mathcal{C}: g(x, y)=0$ are, the more the computing time is. But we can not estimate the singularities of the $g(x, y)=0$.

\section{Conclusion}

In this paper, we present an effective algorithm to determine the topology of a given implicit algebraic surface. The basic idea is to project the surface to plane, obtain the topology information of the plane curve, lift the curve up and determine the space curve segments on the surface, compose surface patches by space curve segments. Experiments show our algorithm works effectively.

\section{References}

[1] Arnborg, S. and Feng, H., Algebraic decomposition of regular curves. J. Symbolic Comput. $5(1,2)(1988): 131-140$

[2] Arnon, D. S., Collins, G. and McCallum S., Cylindrical algebraic decomposition I: the basic algorithm. Quantifier Elimination and Cylindrical Algebraic Decomposition, Edited by B. Buchberger and G. E. Collins, Springer

[3] Arnon, D. S. and McCallum, S., A polynomial-time algorithm for the topological type of a real algebraic curve, J. Symbolic Comput. 5(1,2)(1988): 213-236

[4] Bajaj, C., Hoffmann, C.M., Lynch, R.E. and Hopcroft, J.E.H., Tracing surface intersection, Computer Aided Geometric Design (1988)5: 285-307

[5] Feng, H., Decomposition and computation of the topoplogy of plane real algebraic curves, $\mathrm{PhD}$ Thesis. (1992) The Royal Institute of Technology, Stockholm, Sweden.

[6] Fortuna, E., Gianni, P., Parenti, P. and Traverso, algorithms to compute the topology of orientable real algebraic surfaces, Journal of Symbolic Computation, 36(2003)343-364

[7] Gao, X.S. and Li, M., Rational Quadratic Approximation to Real Algebraic Curves, Computer Aided Geometric Design (2004)21: 805-828 
[8] Gonzalez-Vega, L. and El Kahoui, M., An improve upper complexity bound for the topology computation of a real algebraic plane curve. J. Complexity (1996)12: 527-544

[9] Gonzalez-Vega, L. and Necula, I.. Efficient topology determination of implicitly defined algebraic place curves, Computer Aided Geometric Design, 19(2002): 719-743

[10] Hong, H., An efficient method for analyzing the topology of plane real algebraic curves. Math. Comput. Simulation (1996) 42(4-6): 571-582

[11] Keyser, J., Culver, T. and Krishnan, S., Efficient and exact manipulation of algebraic points and curve. Computer Aided Design (2000) 32(11): 649-662

[12] Sakkalis, T., The topological configuration of a real algebraic curve. Bull. Australian Math. Soc. (1991) 43(1): 37-50

[13] Walker, R. J., Algebraic curves, Springer-Verlag(1978)

[14] W.T. Wu Mathematics Mechanization, Sience Press/Kluwer, Beijing, 2000. 